\title{
Study of Accessory Head of Flexor Pollicis Longus in South Indian Population
}

\author{
Dr.S.Bharathi Rani ${ }^{1}$, Dr.N.S.Sunitha ${ }^{2}$, Dr.J.Karpagajothi ${ }^{3}$ \\ (Assistant professors, Institute of anatomy, Madurai medical college, The Tamilnadu Dr.M.G.R Medical \\ university, Tamilnadu, India).
}

\begin{abstract}
:
Background and Aim: Flexor pollicis longus (FPL) is one of the deep muscles of the flexor compartment of forearm, which is comparatively a recent acquisition in the evolution of upper limb muscle. According to literatures accessory head of FPL (AHFPL) is be found in more than 50\% of individuals worldwide. The present study is taken with the aim to show the incidence, morphology, relation of AHFPL with median and anterior interosseous nerve in south Indian population.

Materials and methods: The study was carried out in 50 upper limb of 25 cadavers in the Department of Anatomy, Madurai Medical college. Dissection was done by volar incision from the distal arm extending into the palm. Results: AHFPL was found in 12 cadavers (48\%), out of which it was bilateral in 10 cadavers (40\%) and unilateral in 2 cadavers(8\%). All AHFPL originated from the medial epicondyle of humerus and inserted into the Proximal 1/3 of FPL tendon. The average length of muscle belly of AHFPL was $6.05 \mathrm{cms}$ and that of the tendon was found to be $3.12 \mathrm{cms}$ bilateraly. Majority (90.9\%) of the AHFPL were supplied by Anterior interosseous nerve. AHFPL was found between Median nerve and AIN in all the cases.

Conclusion: Anatomical Knowledge of AHFPL is useful for the diagnosis and surgical intervention of nerve entrapment syndromes like anterior interosseous nerve syndrome
\end{abstract}

Key Words: Flexor pollicis longus, Accessory head, Anterior interosseous nerve

\section{Introduction}

Flexor pollicis longus (FPL) is one of the deep muscles of the flexor compartment of forearm. FPL arises from the grooved anterior surface of body of radius and also from adjacent interosseous membrane. It may have an additional head which originates from the lateral border or rarely medial border of the coronoid process of the ulna or from the medial epicondyle of humerus [1] which has been called as Gantzer muscle. FPL courses downwards to cover the entire width of the anterior surface of the radius and the tendon passes behind the flexor retinacula. Finally, it inserts into the palmar surface of the base of the distal phalanx of the thumb. Accessory head of Flexor pollicis longus(AHFPL) muscle runs obliquely downwards from medial to lateral side underneath the Flexor digitorum superficialis (FDS) and joins with the FPL muscle [1]. FPL is supplied by anterior interosseous nerve(AIN) which descends on the interosseous membrane between FPL and Flexor digitorum profundus (FDP).It courses downwards to supply the deep flexors of the forearm namely FPL, Flexor digitorum profundus and Pronator quadratus. Anterior Interosseous nerve Syndrome, a rare clinical entity can be caused by the compression of anterior interosseous nerve by any of the several structures present in the forearm. AHFPL, if present could be a causative factor for this syndrome. Knowledge of the morphology of AHFPL is useful for the diagnosis and surgical intervention of nerve entrapment syndromes like anterior interosseous nerve syndrome, rarely carpal tunnel syndrome.

\section{Materials And Methods}

The study was carried out in 50 upper limbs of 25 cadavers in the Department of Anatomy, Madurai Medical college, Madurai. Dissection was done using volar incision from the distal arm extending into the palm. AHPL was observed and it was studied with regard to the following parameters:

*Incidence

*Unilateral or Bilateral variant

*Study of morphology - origin, insertion, length, nerve supply

*Relation with Median nerve and AIN.

\section{Results}

AHFPL was found in 12 cadavers ( 48\% ), out of which it was bilateral in 10 cadavers (40\%) and unilateral in 2 cadavers $(8 \%)$ as shown in fig 1 . In one of the cadavers we found another accessory belly for Flexor Digitorum Profundus along with accessory head of FPL. The morphological features of AHFPL are tabulated in table 1 . 


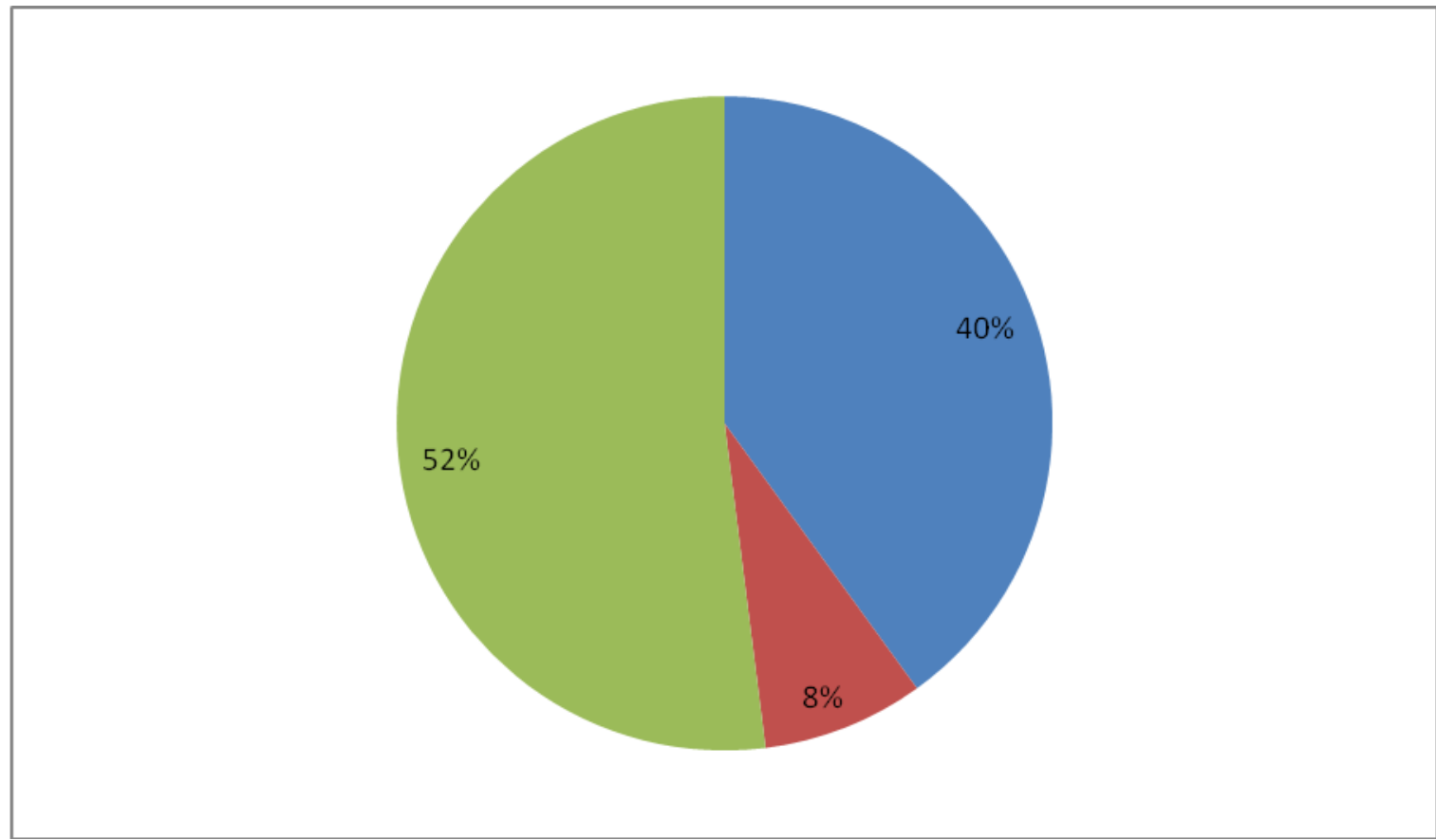

fig 1 pie diagram showing incidence of AHFPL ( in Percentages)

TABLE :1 Morphological Features of Accessory Head of Fpl And Fdp

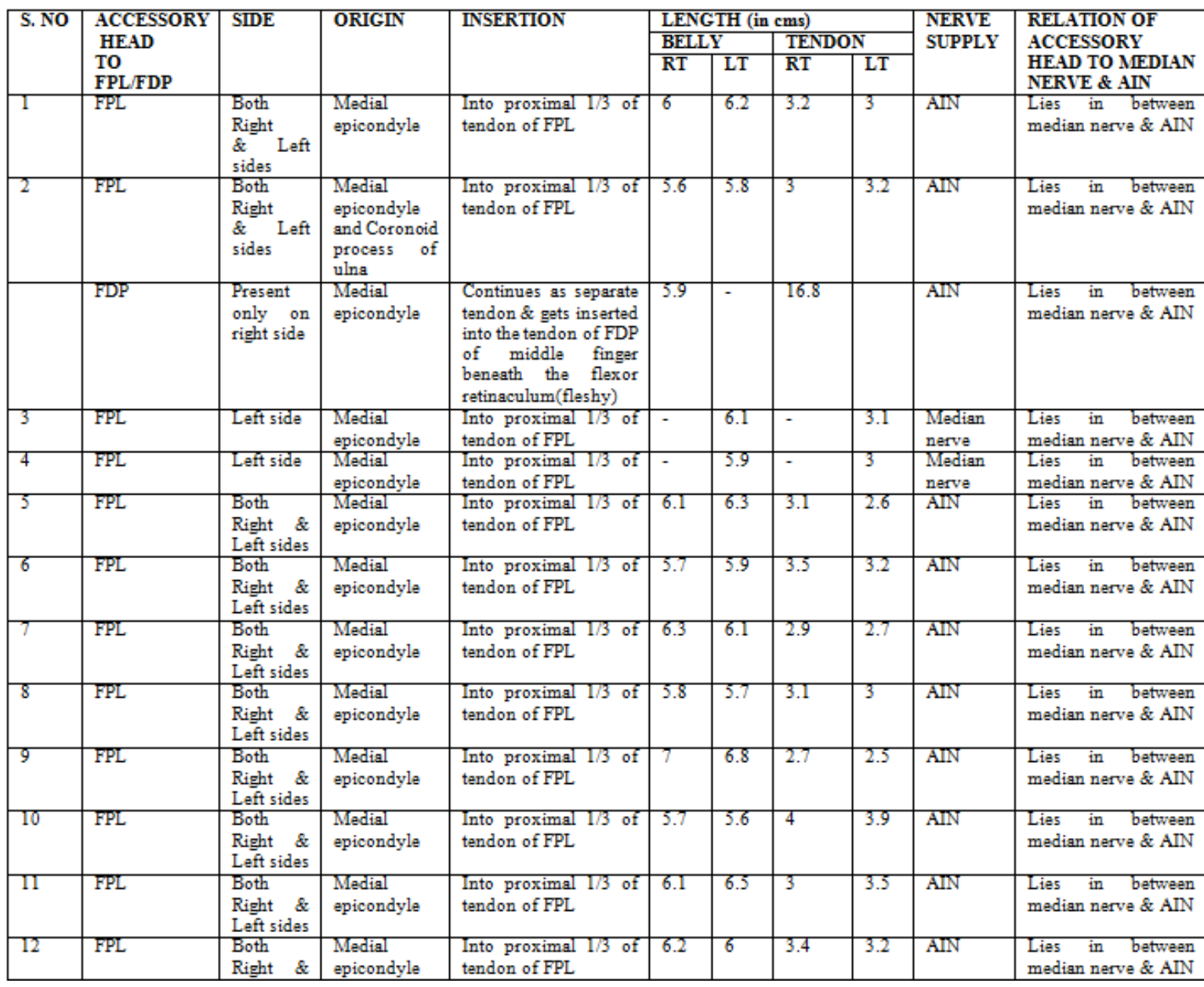

All Accessory heads of FPL originated from the medial epicondyle of humerus underneath FDS and one had few fibres from coronoid process of ulna.AHFPL gets inserted into the Proximal 1/3 of tendon of FPL . 
The shape of the accessory heads was fusiform in nature. The average length of muscle belly of AHPL on the right side was found to be $6.05 \mathrm{cms}$ and on the left was $6.07 \mathrm{cms}$. The average length of the tendon of AHPL on the right side was found to be $3.19 \mathrm{cms}$ and on the left was $3.07 \mathrm{cms}$.

Out of the 22 AHPL, 20 were supplied by Anterior interosseous nerve (90.90\%), and the rest two (9.09\%) were supplied by a branch from the median nerve. AHPL was found between Median and AIN in all the cases. Lateral branch of AIN to FPL is lateral to AHPL whereas medial branch of AIN to FDP lies deep to the AHPL and supplies FDP (fig 2).

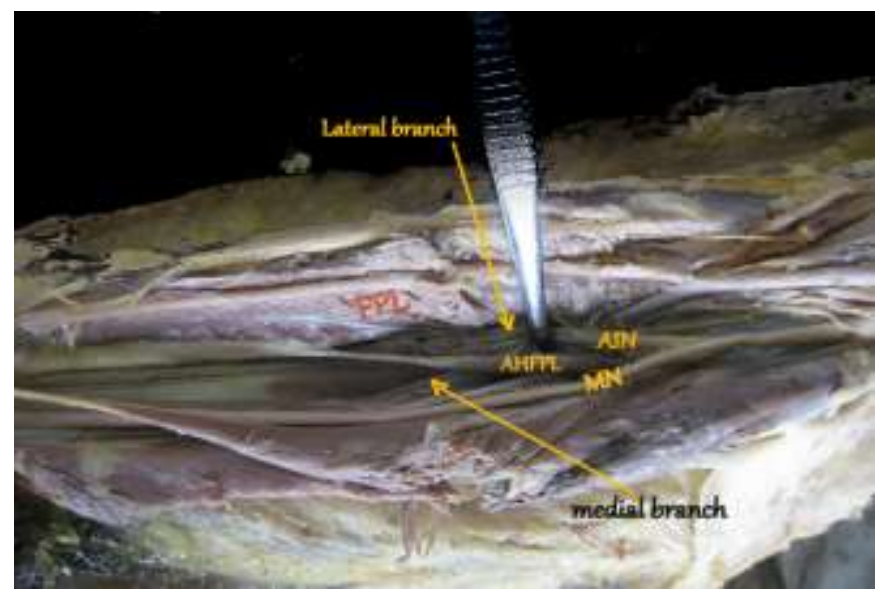

FIG 2 : FPL - Flexor Pollicis Longus, AHFPL - Accessory head of FPL, MN -Median Nerve, AIN - Anterior Interosseus Nerve

Accessory head of FDP originated from the medial epicondyle of humerus from the deep surface of FDS. The belly length was about $5.9 \mathrm{~cm}$, then it ran as a slendor tendon of about $16.8 \mathrm{~cm}$ on the lateral aspect of FDP and inserted as a fleshy part into the tendon of FDP of middle finger beneath the flexor retinaculum (fig 2,3).

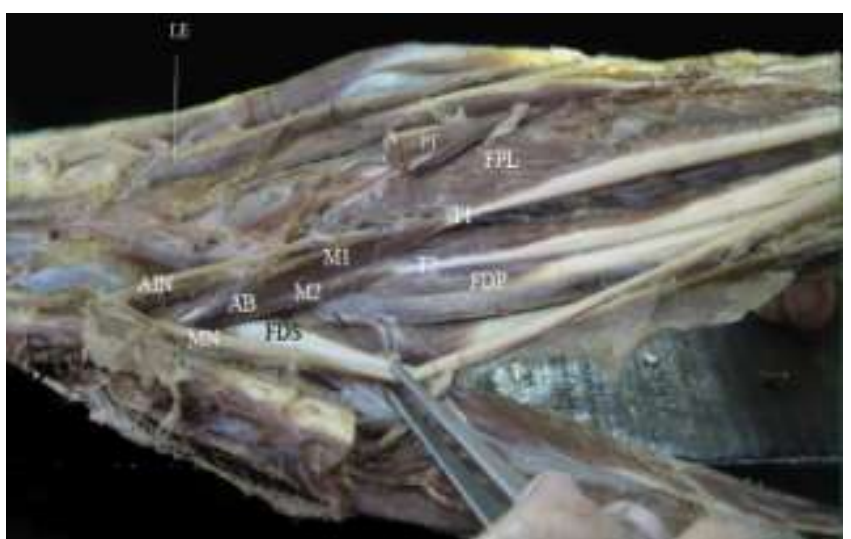

FIG 3 : AB -Accessory belly, FPL - Flexor Pollicis Longus ,FDP - Flexor Digitorum Profundus, M1 - Accessory belly of FPL, M2 - Accessory belly of FDP, T1 - Tendon of FPL, T2 - Tendon of FDP , MN -Median Nerve, AIN - Anterior Interosseus Nerve 


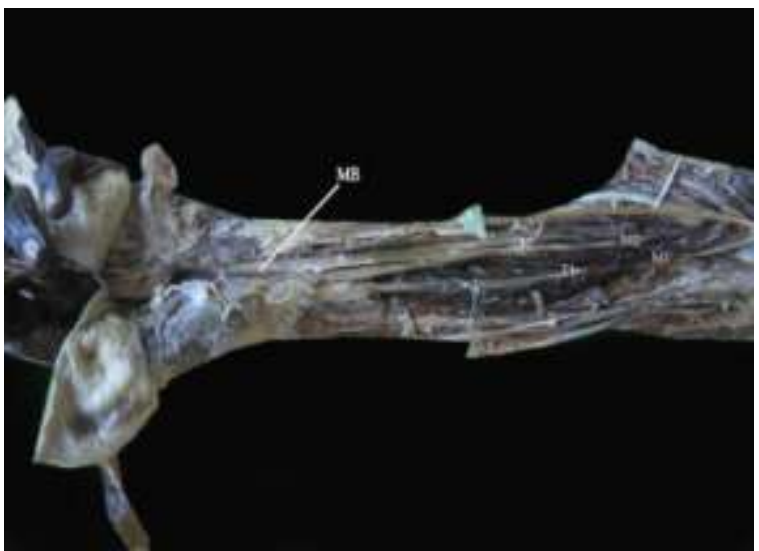

FIG 4 : M1 - Accessory belly of FPL, M2 - Accessory belly of FDP,T1 - Tendon of FPL,

T1 - Tendon of FDP , MB -fleshy part of accessory FDP TENDON

\section{Discussion}

FPL muscle is comparatively a recent acquisition in the evolution of upper limb muscle. Looking back into the transformation and changes occurred during the evolution of upper limb muscle, FPL was absent in primates such as Gorillas and Chimpanzees which had FHL (flexor hallucis longus) to perform the same function done by FPL in humans. It is also evident that in primates, tendon similar to FPL was first evolved with same site of insertion but differing in their origin. Later FPL was found as a bifurcation from Flexor digitorum profundus. Thus anatomical variation of FPL can be explained as a result of retention of traits in humans reflecting its anatomy of his ancestors. In the present study, the incidence of AHPL is found to be $48 \%$, which is closer to the study made by Dellon [2] and Al Qattan [3] but differs from Mangini U [4], Oh et al [5] and Hemmady [6]. Based on the literature, it was observed that AHFPL could be present in more than $50 \%$ of individuals worldwide.

TABLE 2: Comparision of Incidence of AHPL with Previous Studies

\begin{tabular}{|l|l|l|}
\hline S NO & STUDIES & INCIDENCE (\%) \\
\hline 1 & Present study & 48 \\
\hline 2 & Mangini (1960) & 73.7 \\
\hline 3 & Al Qattan & 52 \\
\hline 4 & Dellon & 45 \\
\hline 5 & Hemmady & $66.6 \%$ \\
\hline 6 & Oh & $66.7 \%$ \\
\hline 7 & Mahakkannukrauh & $62.1 \%$ \\
\hline 8 & Jones & $55 \%$ \\
\hline
\end{tabular}

Bilateral variant was reported to be common in the study made by Jones and Abrahams [7] (1997) and Hemmady et al [6]. In the present study also we found that the bilateral variant was common (48\%). All the AHFPL in the study originated from the medial epicondyle of humerus .Of these one had combined origin from medial epicondyle and coronoid process similar to the studies of Wood [8] (1868), Macalister [9] (1875), Malhotra et al [10] . Regarding the insertion, all the AHFPL of the present study was found to be inserted into the proximal third of the tendon of FPL and thus appeared to be a continuation of the main FPL tendon as observed by Mangini U [4] 1960.

Fusiform shape of AHFPL was found to be common in the present study. Fusiform muscle produces quick and wide range of movement in opposition to the the pennate FPL muscle which produces high force for smaller range of motion. According to Levanjee and Norkin [11], the entire muscle determines its function and range of movement. This would cause strain over the normal range of movement and in turn there would be loss of precise and skillfull movement.

The average length of muscle belly and the tendon length coincided with the studies made by Jones M \& Abrahams [7], Potu B.k et al [12].

According to Mahakkanukrauh P et al [13] 4 types of relationships of AHFPL to AIN were noted.

1 - AIN running anterior to AHFPL

2 - AIN running lateral to AHFPL

3 - AIN running posterior to AHFPL

4 - AIN running lateral and posterior to AHFPL

TYPE 3 and 4 were associated with AIN syndrome. Type 3 relationship would lead to a complete AINS as the AIN passes posterior to accessory head producing weakness of FPL, FDP of index and middle 
fingers and the pronator quadratus muscles. Incomplete AINS was produced when medial branch of AIN to FDP alone is compressed beneath the accessory head (Type 4 relation). The present study observed a type 4 relationship between AIN and AHFPL as noted by Tabib et al [14], where medial branch of AIN to FDP passes posterior to the AHFPL, can be compressed while the lateral branch to FPL won't be affected .

AIN syndrome is manifested as weakness or motor loss of FPL, FDP to index finger, pronator quadratus and occasionally the FDP to the middle finger. In a AINS, the weakness or loss of motor function occur spontaneously. Patients describe clumsiness with fine motor skills such as writing and pinching. As AIN does not innervate the skin, the syndrome is not associated with sensory loss. Accessory muscle in the forearm causes compression of neurovascular structures . Degreef and Desmet (2004) [15] reported a case of paralysis of AIN due to accessory muscle compressing the nerve. According to Kaplan [16], injury to AHFPL produces secondary contracture of thumb resulting in check reign effect in thumb. Hence this variant has to be kept in mind by the surgeons while carrying out an anterior approach in the forearm. Mangini $U$ [4] reported a prevalence of accessory head of FDP (AHFDP) of $2.7 \%$, which coincided with the present study where accessory head was observed in one limb (2\%). The AHFDP of present study had its origin from the deep surface of FDS similar to Macalister [9] (1875) and Wood [8] (1868).

Macalister [9] described insertion of AHFDP in 9 possible ways. The present study described insertion into middle finger tendon.

Eintin [17] grouped the causes of carpal tunnel syndrome into 3 categories

I- $\quad$ Those reducing the capacity of the tunnel

II- Those increasing the volume of its contents

III- Those forming a part of systemic condition.

In the present study, accessory head of FDP inserted as a fleshy part into FDP tendon of middle finger, beneath flexor retinaculum increase the volume of content of carpal tunnel causing carpal tunnel syndrome.

\section{Conclusion}

Presence of accessory heads or muscles should be borne in mind by the surgeons as causative factors for neuropathies and compressive effects. These variations may simulate a soft tissue mass and may lead to misinterpretation. Hence anatomical knowledge of these variation will be of help to radiologists and orthopaedicians.

\section{References}

[1]. Standring.S, Borley NR et al. Gray's anatomy: The Anatomical basis of clinical practice. $40^{\text {th }}$ edition. Elsevier Ltd.London,2008 : Ch $49: \mathrm{p}-848$

[2]. Dellon AL, Mackinnon SE. Musculo aponeurotic variations along the course of the median nerve in the Proximal forearm. J Hand Surg Br. 1987; 12: 359-363.

[3]. Al-Qattan MM. Gantzer's muscle. An anatomical study of the accessory head of the flexor pollicis longus muscle. J Hand Surg Br. 1996; $275: 269-270$.

[4]. Mangini U. Flexor pollicis longus muscle. Its morphology and clinical significance. J Bone Joint Surg Am. 1960; 42: 467-470.

[5]. Oh CS, Chung IH, Koh KS. Anatomical study of the accessory head of the flexor pollicis longus and the anterior interosseus nerve in Asians. Clin Anat. $2000 ; 13: 434-438$.

[6]. Hemmady MV, Subramanya AV, Mehta IM. Occasional head of flexor pollicis longus muscle: a study of its morphology and clinical significance. J Postgrad Med. 1993; 39: 14-16.

[7]. Jones M, Abrahams PH, Sanudo JR, Campillo M. Incidence and morphology of accessory heads of flexor pollicis longus and flexor digitorum profundus (Gantzer's muscle). J Anat. 1997; 191: 451-455.

[8]. Wood J. Variations in human myology. Proceedings of the Royal Society of London 1868; 16: 483-525.

[9]. Macalister A.Additional observations on muscular anomalies in human anatomy (3rd series), with a catalogue of the principal muscular variations hitherto published.Transactions of the Royal Irish Academy. $1875 ; 25: 1-134$.

[10]. Malhotra VK, Sing NP, Tewari SP. The accessory head of the flexor pollicis longus muscle and its nerve supply. Anat Anz. 1982; 151: 503-505

[11]. Levangie PK, Norkin CC. Joint Structure and Function: A Comprehensive Analysis. 4th Ed., Philadelpia, F. A. Davis Company. $2006 ; 120-121$.

[12]. Potu B K, Gorantla V R, Pulakunta T, Rao M S, Mamatha T, Vollala V R, Nayak S R ,Accessory head of flexor pollicis longusmuscle and its significance in anterior interosseus nerve syndrome :Case report and review . Int . J.Morphol 25(4): 911 -914, 2007

[13]. Mahakkanukrauh P, Surin P, Ongkana N, Sethadavit M, Vaidhayakarn P. Prevalence of accessory head of flexor pollicis longus muscle and its relation to anterior interosseus nerve in Thai population. Clin Anat.2004; 17: 631-635.

[14]. Tabib W, Aboufarah F ,Asselineau A. Compression of anterior interosseus nerve by Gantzer's muscle. Chir Main , 20: $241-6$ ,2001.

[15]. Degreef I, Desmet L. Anterior interosseous nerve paralysis due to gantzers muscle Acta orthopaedica Belgica 2004; 70: 482-484

[16]. Kaplan EB. Correction of a disabling contracture of the thumb. Bull Hosp Joint Dis. 1942; 3: 51-54.

[17]. Entin MA: Carpal tunnel syndrome and its variants. Surg Clin North Am 1968, 48:1097- 1112. 